\title{
Surface reconstruction transition of metals induced by molecular adsorption
}

\author{
J. T. Sun, ${ }^{1}$ L. Gao, ${ }^{1}$ X. B. He, ${ }^{1}$ Z. H. Cheng, ${ }^{1}$ Z. T. Deng, ${ }^{1}$ X. Lin, ${ }^{1}$ H. Hu, ${ }^{1}$ S. X. Du, ${ }^{1}$ Feng Liu, ${ }^{2}$ and H.-J. Gao ${ }^{1, *}$ \\ ${ }^{1}$ Institute of Physics, Chinese Academy of Sciences, P. O. Box 603, Beijing 100190, China \\ ${ }^{2}$ Department of Materials Science and Engineering, University of Utah, Salt Lake City, Utah 84112, USA
}

(Received 1 December 2010; published 11 March 2011)

\begin{abstract}
Two surface reconstruction patterns, strikingly different from the $\mathrm{Au}(111)$ intrinsic herringbone reconstruction, have been observed at the interfaces between $\mathrm{Au}(111)$ and the self-assembled monolayer of perylene or iron phthalocyanine by scanning tunneling microscopy (STM). These interfacial structures, elucidated by using the two-dimensional Frenkel-Kontorova model and density-functional theory (DFT) calculations, are attributed to the anisotropic surface stress induced by interfacial charge transfer. The length scales of the induced reconstruction periodicities are theoretically estimated, in quantitative agreement with experiments. Our findings afford the possibility of tailoring the contact structure of molecular electronic devices by molecular and surface engineering.
\end{abstract}

DOI: 10.1103/PhysRevB.83.115419

PACS number(s): 85.65.+h, 68.35.-p, 68.37.Ef, 73.40.-c

\section{INTRODUCTION}

Interface is one of the key factors governing the transport properties and performance of molecular devices. ${ }^{1,2}$ The surface reconstruction characterized by the periodic bulk lattice structure often results in surface stress, ${ }^{3-5}$ simultaneously, external adsorbates can modify substrate surface stress, leading to new interface structure or imposing strain onto the epitaxial overlayers. ${ }^{5-7}$ In particular, molecular adsorption induced modification of surface reconstruction is of great importance in that atomic-scale structural variations at molecule-electrode contacts significantly change charge transport, ${ }^{7-10}$ so controlling the coupling between molecules and electrodes with atomic precision is of both fundamental interest and practical importance. ${ }^{11}$

Gold is the most important electrode material in molecular electronics. ${ }^{1-13} \mathrm{Au}(111)$ normally exhibits a $22 \times \sqrt{3}$ surface reconstruction ${ }^{14}$ resulting from the spontaneous formation of "stress domains." 15 Previous studies on Au(111) showed that the adsorption-induced surface stress can change the original $22 \times \sqrt{3}$ reconstruction. ${ }^{16-18}$ While some studies have discussed the mechanisms for the formation of herringbone reconstruction on a bare surface ${ }^{15}$ and the lifting of herringbone reconstruction by alkanethiolate monolayers, ${ }^{19}$ a quantitative description of the molecular adsorption dependent metal surface reconstructions has not been studied in depth.

In this work, we report two surface reconstruction patterns that were experimentally observed on $\mathrm{Au}(111)$, induced by the adsorption of perylene and iron phthalocyanine (FePc) monolayers, which are analyzed with the Frenkel-Kontorova (FK) model and density-functional theory (DFT) calculations. Our calculated length scale of these reconstruction patterns is consistent with scanning tunneling microscopy (STM) observations. We suggest that the difference in interaction strength reflected by charge transfer leads to different stress anisotropy in different molecular adsorption, leading to the observed molecular dependence of reconstruction patterns.

\section{EXPERIMENTS}

Our experiments were performed with an Omicron molecular beam epitaxy low-temperature STM (MBE-LTSTM) system with a base pressure below $3.0 \times 10^{-10}$ mbar. An atomically clean $\mathrm{Au}(111)$ surface was prepared by repeated cycles of $\mathrm{Ar}^{+}$sputtering and subsequent annealing. Highly ordered self-assembled monolayers (SAMs) of perylene and FePc were fabricated by thermal evaporation. ${ }^{9,20}$ The detailed information on these simulations is described in Ref. 21.

\section{RESULTS AND DISCUSSION}

The $\mathrm{Au}(111)$ reconstruction patterns are observed to change upon molecular adsorption of perylene and FePc. Figure 1(a) shows the characteristic herringbone reconstructions of the clean $\mathrm{Au}(111)$ surface, which is composed of alternate facecentered-cubic (fcc) and hexagonal-close-packed (hcp) domains separated by bright corrugation lines. These corrugation lines bend by about $120^{\circ}$ periodically around the domain wall with a periodicity of $200-280 \AA$ in the [1ㅣㅣ direction, as shown in the zoom-in image in Fig. 1(d). Figure 1(b) shows the reconstruction pattern for perylene SAM on $\mathrm{Au}(111)$ with a corrugation height modulation of about $0.2 \AA$. As shown in the zoom-in image in Fig. 1(e), the periodicities of the perylene SAM on $\mathrm{Au}(111)$ become $110 \pm 10 \AA$ in the [1 $\overline{1} 0]$ direction and remain the same in the [112] direction, as in the clean surface. The most distinct transition pattern is observed upon monolayer FePc adsorption on $\mathrm{Au}(111)$, as shown in Fig. 1(c). The long-range height undulation across the surface is about $0.4 \AA$. As shown in Fig. 1(f), the periodicities in $\mathrm{FePc} / \mathrm{Au}(111)$ are about $70 \pm 5 \AA$ in the [110] direction and also remain the same in the [112] direction. The reconstruction pattern transitions from clean to perylene-adsorbed and to FePc-adsorbed $\mathrm{Au}(111)$ surface are schematically depicted in Figs. 1(g), 1(h), and 1(i), respectively. ${ }^{20,22}$

The main difference between the herringbone reconstruction pattern and these two molecular modified reconstruction patterns is the periodicity of the zigzag pattern in the [1 $\overline{1} 0]$ direction. These new reconstruction patterns are believed to form in response to the modification of surface stress induced by molecular adsorption. When a clean gold surface is adsorbed with molecules, the surface electrons respond to the presence of surface adsorbates, and hence the charge distribution near the surface becomes different from that before molecular adsorption. The adsorption induced surface stress is a consequence of this redistribution of electronic charge. ${ }^{4}$ Local surface stress relief is the most likely driving force for 

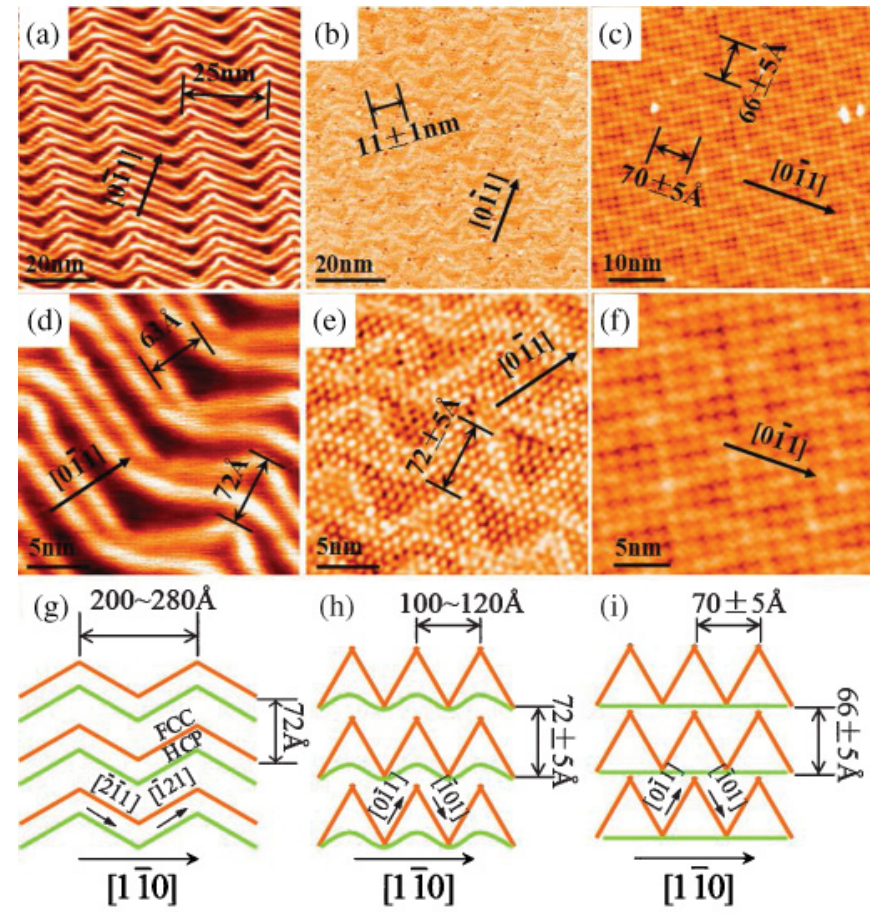

FIG. 1. (Color online) (a)-(f) STM images showing surface reconstruction patterns for the clean $\mathrm{Au}(111)$ surface [(a) and (d)], perylene/Au(111) [(b) and (e)], and $\mathrm{FePc} / \mathrm{Au}(111)$ [(c) and (f)]. Image sizes: $100 \mathrm{~nm} \times 100 \mathrm{~nm}$ for (a) and (b); $50 \mathrm{~nm} \times 50 \mathrm{~nm}$ for (c); $28 \mathrm{~nm} \times 28 \mathrm{~nm}$ for $(\mathrm{d}),(\mathrm{e})$, and (f). Scanning parameters: $U=$ $-1.2 \mathrm{~V}, I=0.05 \mathrm{nA}$. The molecular appearance for perylene and $\mathrm{FePc}$ on the $\mathrm{Au}(111)$ surface is shown as bright spots and cross shape in (e) and (f), respectively. (g)-(i) Sketches of surface reconstruction patterns for the clean $\mathrm{Au}(111)$ surface (g), perylene/Au(111) (h), and $\mathrm{FePc} / \mathrm{Au}(111)$ (i).

the new surface reconstructions on $\mathrm{Au}(111) .{ }^{19,23}$ To confirm our hypothesis, we performed first-principles calculations to estimate the molecular adsorption induced surface stress.

Several possible configurations have been considered for perylene and FePc molecules on $\mathrm{Au}(111)$, as shown in Fig. 2. For both molecules at monolayer coverage, the bridge site was found to be the most stable adsorption site on $\mathrm{Au}(111) .^{20}$ Figure 2(b) is the side view of the most stable configurations. The perylene molecule is tilted along one direction lying out-of-plane on the surface, which is consistent with high resolution STM images. ${ }^{20}$ The molecular center of FePc points downward slightly; and the two $\mathrm{Au}$ atoms underneath the $\mathrm{Fe}$ atom are lifted by $0.2 \AA$. The shortest distance between the adsorbed molecule and the Au substrate is about $3.21 \AA$ for perylene and $2.94 \AA$ for FePc, respectively, which implies that the molecule-substrate interaction of $\mathrm{FePc} / \mathrm{Au}(111)$ is stronger than that of perylene/ $\mathrm{Au}(111)$.

Based on the most stable configurations, we calculated the surface stress anisotropy of both adsorption systems along the perpendicular direction as shown in the upper left part of Fig. 2(a). The surface stress anisotropy is obtained as $\Delta \sigma^{s}=\sigma_{\perp}^{s}-\sigma_{\|}^{s}$, where $\sigma_{\perp}^{s}$ and $\sigma_{\|}^{s}$ denote the surface stress component along the nearest-neighbor direction [011] and the next-nearest-neighbor direction [121], respectively. ${ }^{21}$ Our calculations show that the surface stress anisotropy is about

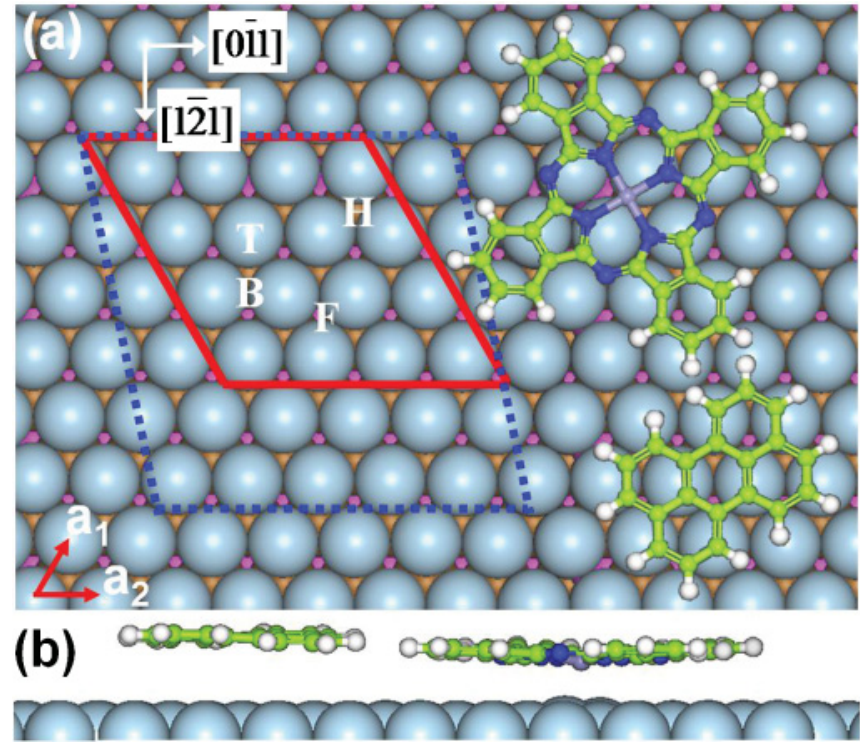

FIG. 2. (Color online) (a) Top view and (b) side view of the most stable adsorption configurations of perylene and FePc molecules on $\mathrm{Au}(111)$ at monolayer coverage. Red solid (blue dashed) line corresponds to the experimental observed matrix for perylene $(\mathrm{FePc})$ on the $\mathrm{Au}(111)$ surface (Refs. 20-22). One molecular orientation and four adsorption sites are considered for both molecules. T, H, F, and B labels represent top, hcp, fcc, and bridge sites, respectively.

$5.88 \mathrm{meV} / \AA^{2}$ for perylene $/ \mathrm{Au}(111)$ and $163.56 \mathrm{meV} / \AA^{2}$ for $\mathrm{FePc} / \mathrm{Au}(111)$, with compressive stress along both directions. In contrast, the clean $\mathrm{Au}(111)$ surface has an isotropic tensile stress. ${ }^{4,19}$ The surface stress anisotropy for $\mathrm{FePc} / \mathrm{Au}(111)$ is much larger than that for perylene/Au(111), which indicates that the FePc-induced reconstruction pattern of $\mathrm{Au}(111)$ will be much different from the perylene-induced reconstruction.

To quantify the change in reconstruction pattern, long-range elastic interaction needs to be considered, as the molecular adsorption induced reconstruction transition results from the competition between the domain wall energy (which is due to the spontaneous formation of stress domains) and the longrange elastic relaxation energy. ${ }^{15}$ The elastic relaxation energy induced by surface stress discontinuity decreases the surface free energy, which is derived as ${ }^{15}$

$$
E_{\mathrm{el}}=-\frac{c_{2}}{l} \ln \left(\frac{l}{\pi a_{d}}\right)
$$

Here $c_{2}=\frac{3}{8 \pi} \frac{\Delta \sigma^{2}}{\mu}, \Delta \sigma^{s}=\sigma_{\perp}^{s}-\sigma_{\|}^{s}$ denotes the stress anisotropy, $\sigma_{\perp}^{s}$ and $\sigma_{\|}^{s}$ can be directly obtained from firstprinciples calculations for both systems as illustrated above, $a_{d}$ is the width of the domain wall, and $\mu$ is the shear modulus. Thus, the surface free energy density can be minimized when the length scale of the stress domain pattern adopts ${ }^{15}$

$$
l_{0}=\pi a_{d} e^{c_{1} / c_{2}+1},
$$

where $c_{1}$ denotes the domain wall energy per unit length, which can be obtained from the FK model. ${ }^{24-27}$ The Hamiltonian of the FK model is

$$
H=\sum_{i} V_{\mathrm{su}}\left(R_{i}\right)+\sum_{i, j} V_{\mathrm{ss}}\left(R_{i}-R_{j}\right),
$$




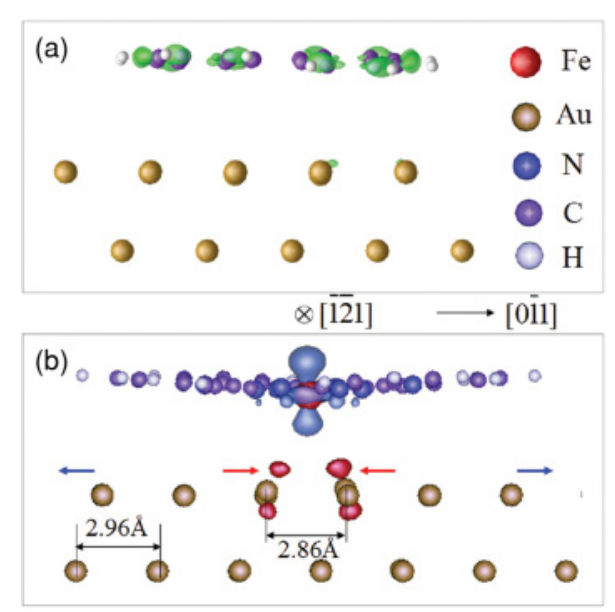

FIG. 3. (Color online) Differential charge density of the most stable configuration of perylene (a) and that of $\mathrm{FePc}(\mathrm{b})$ on $\mathrm{Au}(111)$. The blue and red colors denote charge accumulation and depletion orbitals at iron and gold atoms, respectively. These two panels have the same color denotation. The arrows lying just below the iron atom indicate the reduction of the nearest bond length $2.86 \AA$ along the $[0 \overline{1} 1]$ direction as compared with the bulk bond length $2.96 \AA$.

where $R_{i}=\left(x_{i}, y_{i}\right)$ is the position of the $i$ th atom. The first item, which represents the substrate potential in the FK model, is expanded as a two-dimensional (2D) Fourier series in reciprocal space. In the second item, the Morse potential is employed to describe the $2 \mathrm{D}$ surface potential. ${ }^{25}$

The 2D FK model was completely parametrized from firstprinciples calculations. ${ }^{20-24}$ After obtaining the Hamiltonian of the FK model, the steepest descent and conjugated gradient procedures were employed to minimize the total energy of the unreconstructed $\mathrm{Au}(111)$ surface with adsorbates. Then we obtained the energy per unit length of each domain wall as $c_{1}=0.95 \pm 0.05 \mathrm{meV}$ for perylene $/ \mathrm{Au}(111)$ and $c_{1}=0.55 \pm$ $0.05 \mathrm{meV}$ for $\mathrm{FePc} / \mathrm{Au}(111)$, respectively. After examining the width of the domain wall, we use $a_{d}=12.0 \pm 2.0 \AA$. Upon substituting $c_{1}, c_{2}$, and $a_{d}$ into Eq. (2), we obtain the length scale of the reconstruction patterns with a periodicity in the [110] direction of 71.1-98.3 $\AA$ for perylene/Au(111) and 59.1-81.5 $\AA$ for $\mathrm{FePc} / \mathrm{Au}(111)$, respectively. These results are consistent with our STM observations of $110 \pm 10 \AA$ for perylene/Au(111) and $70 \pm 5 \AA$ for $\mathrm{FePc} / \mathrm{Au}(111)$, respectively, as shown in Fig. 1. According to Eq. (2), the length scale of the reconstruction pattern is determined by two parameters, $c_{1}$ and $c_{2}$. Our DFT calculations show only small variations in $c_{1}$, which indicates that the different quantity in $c_{2}$ plays the major role in determining the length scales of the two reconstruction patterns, which scale exponentially in an inverse manner with the stress anisotropy at the domain boundary. As shown earlier by DFT calculations, the stress anisotropy in perylene/Au(111) is much smaller than that in $\mathrm{FePc} / \mathrm{Au}(111)$. Consequently, the length scale of the reconstruction pattern in perylene/ $\mathrm{Au}(111)$ is longer than that in $\mathrm{FePc} / \mathrm{Au}(111)$.

The change of the long-range reconstruction pattern has an electronic origin. The interaction between perylene molecules and the Au substrate occurs mainly through the conjugated

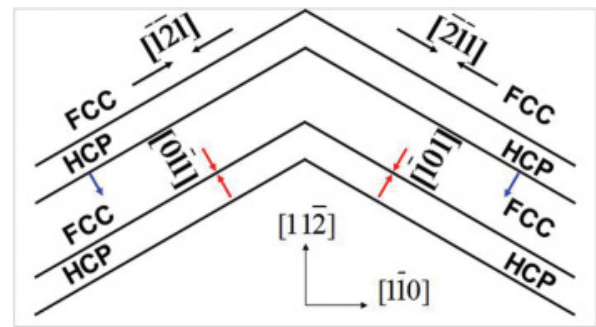

FIG. 4. (Color online) Schematic representation of the anisotropic stress distribution of the $\mathrm{Au}(111)$ surface upon molecular adsorption.

rings. As a result, there is only a small charge transfer between perylene and the $\mathrm{Au}(111)$ surface except for a slight charge redistribution within the molecular plane. The slight nonuniform charge redistribution in the perylene-adsorbed $\mathrm{Au}(111)$ surface would not disturb the hexagonal symmetry of the underlying surface substantially. In contrast, the interaction between FePc molecules and the Au substrate occurs mainly through the coupling between the center Fe atom and the two $\mathrm{Au}$ atoms at the bridge site, as shown in Fig. 3. The strong coupling between $\mathrm{FePc}$ and the $\mathrm{Au}(111)$ surface makes the nearest-neighbor bridge-site gold atoms closer to each other by $0.1 \AA$ as compared to the bulk bond length [Fig. 3(b)]. The reduction of the substrate bond length at the moleculeelectrode contact causes a local compressive stress along the [01ㅣ direction, leading to large surface stress anisotropy. This is drastically different from the isotropic tensile stress of the bare $\mathrm{Au}(111)$ surface, as indicated by red arrows in Fig. 4. On the other hand, if one FePc molecule is lying above the bridge site locally, the other part along the [011] direction will be smoothed spontaneously (blue arrows in Fig. 4). Furthermore, the compressive stress along the [1 $\overline{2} 1]$ direction for both kinds of adsorbates causes the reduction in the [011] direction as well. Although the perylene molecules are sitting above the bridge site of the substrate, the effect of local stress relief is not as strong as that of FePc on the $\mathrm{Au}(111)$ surface, preserving the hexagonal symmetry of the $\mathrm{Au}(111)$ substrate. In comparison, the much stronger interaction in $\mathrm{FePc} / \mathrm{Au}(111)$ leads to more dramatic redistribution of the electronic charge near the $\mathrm{Au}$ surface, resulting in a larger surface stress anisotropy that drives the herringbone reconstruction into a far different reconstruction pattern from the starting one.

\section{CONCLUSIONS}

In summary, two comparative surface reconstruction patterns on $\mathrm{Au}(111)$ induced by the adsorption of perylene and FePc SAMs have been observed by STM experiments and illustrated using the FK model and DFT calculations. The change of surface stress anisotropy induced by different amounts of charge transfer is shown to be responsible for the formation of molecular adsorption dependent reconstruction patterns. Our findings shed some new light on the nature and strength of molecule-metal interface interactions, and on molecular and surface engineering of the molecule-electrode contact in molecular electronics, where interface structures and properties are expected to be the dominating factors in determining interfacial electronic transport. 


\section{ACKNOWLEDGMENTS}

This project was partially supported by the Natural Science Foundation of China, CNIC, CAS, and SSC. We are also grateful to Professor Narasimhan and Dr. Pushpa (JNCASR, Bangalore, India) for generously sharing their source code with us. *hjgao@iphy.ac.cn

${ }^{1}$ R. S. Jacobsen, K. N. Andersen, P. I. Borel, J. Fage-Pedersen, L. H. Frandsen, O. Hansen, M. Kristensen, A. V. Lavrinenko, G. Moulin, H. Ou, C. Peucheret, B. Zsigri, and A. Bjarklev, Nature (London) 441, 199 (2006).

${ }^{2}$ M. Hÿtch, F. Houdellier, F. Hüe, and E. Snoeck, Nature (London) 453, 1086 (2008).

${ }^{3}$ M. Gsell, P. Jakob, and D. Menzel, Science 280, 717 (1998).

${ }^{4}$ H. Ibach, Surf. Sci. Rep. 29, 195 (1997).

${ }^{5}$ F. Liu and M. G. Lagally, Phys. Rev. Lett. 76, 3156 (1996).

${ }^{6}$ R. Berger, E. Delamarche, H. P. Lang, C. Gerber, J. K. Gimzewski, E. Meyer, and H.-J. Güntherodt, Science 276, 2021 (1997).

${ }^{7}$ G. V. Nazin, X. H. Qiu, and W. Ho, Science 302, 77 (2003).

${ }^{8}$ Y. B. Hu, Y. Zhu, H.-J. Gao, and H. Guo, Phys. Rev. Lett. 95, 156803 (2005).

${ }^{9}$ L. Gao, W. Ji, Y. B. Hu, Z. H. Cheng, Z. T. Deng, Q. Liu, N. Jiang, X. Lin, W. Guo, S. X. Du, W. A. Hofer, X. C. Xie, and H.-J. Gao, Phys. Rev. Lett. 99, 106402 (2007).

${ }^{10}$ W. W. Pai, H. T. Jeng, C.-M. Cheng, C.-H. Lin, X. Xiao, A. Zhao, X. Zhang, G. Xu, X. Q. Shi, M. A. Van Hove, C.-S. Hsue, and K.-D. Tsuei, Phys. Rev. Lett. 104, 036103 (2010).

${ }^{11}$ A. Nitzan and M. A. Ratner, Science 300, 1384 (2003).

${ }^{12} \mathrm{~S}$. Wu, M. T. González, R. Huber, S. Grunder, M. Mayor, C. Schönenberger, and M. Calame, Nat. Nanotech. 3, 569 (2008).

${ }^{13}$ Y. Wang, N. S. Hush, and J. R. Reimers, Phys. Rev. B 75, 233416 (2007).
${ }^{14}$ J. V. Barth, H. Brune, G. Ertl, and R. J. Behm, Phys. Rev. B 42, 9307 (1990).

${ }^{15}$ S. Narasimhan and D. Vanderbilt, Phys. Rev. Lett. 69, 1564 (1992).

${ }^{16}$ E. I. Altman and R. J. Coltona, Surf. Sci. 279, 49 (1992).

${ }^{17}$ J. K. Gimzewski, S. Modesti, T. David, and R. R. Schlittler, J. Vac. Sci. Technol. B 12, 1942 (1994).

${ }^{18}$ G. E. Poirier, Chem. Rev. 97, 1117 (1997).

${ }^{19}$ V. Srinivasan, G. Cicero, and J. C. Grossman, Phys. Rev. Lett. 101, 185504 (2008)

${ }^{20}$ L. Gao, J. T. Sun, Z. H. Cheng, Z. T. Deng, X. Lin, S. X. Du, and H.-J. Gao, Surf. Sci. 601, 3179 (2007).

${ }^{21}$ See supplemental material at [http://link.aps.org/supplemental/ 10.1103/PhysRevB.83.115419] for calculation details.

${ }^{22}$ Z. H. Cheng, L. Gao, Z. T. Deng, Q. Liu, N. Jiang, X. Lin, X. B. He, S. X. Du, and H.-J. Gao, J. Phys. Chem. C 111, 2656 (2007).

${ }^{23}$ C. E. Bach, M. Giesen, H. Ibach, and T. L. Einstein, Phys. Rev. Lett. 78, 4225 (1997).

${ }^{24}$ M. Mansfield and R. J. Needs, J. Phys.: Condens. Matter 2, 2361 (1990).

${ }^{25}$ R. Pushpa and S. Narasimhan, Phys. Rev. B 67, 205418 (2003).

${ }^{26}$ S. C. Erwin, A. A. Baski, L. J. Whitman, and R. E. Rudd, Phys. Rev. Lett. 83, 1818 (1999).

${ }^{27}$ Ž. Crljen, P. Lazić, D. Šokčević, and R. Brako, Phys. Rev. B 68, 195411 (2003). 